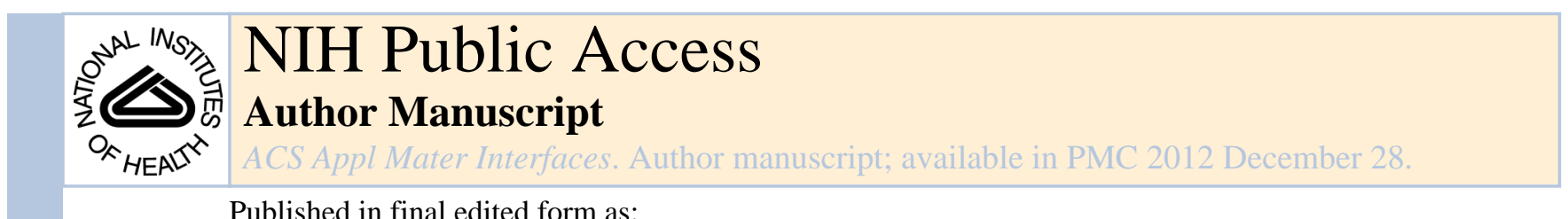

Published in final edited form as:

ACS Appl Mater Interfaces. 2011 December 28; 3(12): 4812-4818. doi:10.1021/am201305x.

\title{
Metal-Mesh Lithography
}

\author{
Zhao Tang, Qingshan Wei, and Alexander Wei \\ Department of Chemistry, 560 Oval Drive, Purdue University, West Lafayette, Indiana \\ 47907-2084
}

\begin{abstract}
Metal-mesh lithography (MML) is a practical hybrid of microcontact printing and capillary force lithography that can be applied over millimeter-sized areas with a high level of uniformity. MML can be achieved by blotting various inks onto substrates through thin copper grids, relying on preferential wetting and capillary interactions between template and substrate for pattern replication. The resulting mesh patterns, which are inverted relative to those produced by stenciling or serigraphy, can be reproduced with low micrometer resolution. MML can be combined with other surface chemistry and lift-off methods to create functional microarrays for diverse applications, such as periodic islands of gold nanorods and patterned corrals for fibroblast cell cultures.
\end{abstract}

\section{Keywords}

lithography; surface chemistry; microarrays; chemisorption; wetting

\section{Introduction}

Surface patterning by lithography is one of the essential tools for micro- and nanoscale technologies. A number of lithographic approaches are able to reproduce features with submicron resolution, including serial writing methods such as electron-beam and scanningprobe lithography 1,2 and photolithography using extreme ultraviolet wavelengths or soft xrays. ${ }^{3,4}$ However, many lithographic applications do not require this level of spatial definition, but are more strongly dependent on the versatility of the patterning method. For this reason, direct-contact printing - arguably the oldest and simplest form of pattern replication- remains one of the most practical and versatile methods of surface patterning. ${ }^{5,6}$ Direct-contact printing mechanisms include mechanical deformation (nanoimprint lithography), ${ }^{9,8}$ anisotropic dewetting induced by capillary forces (capillary force lithography, or CFL), ${ }^{9,10,11}$ and the pressure-mediated deposition of materials using patterned stamps (microcontact printing, or $\mu \mathrm{CP}$ ). ${ }^{12,13,14}$ The latter has become highly popular due to its relative ease of implementation: patterning is readily achieved by casting solutes (inks) onto elastomeric polydimethylsiloxane (PDMS) stamps with appropriate wetting and adhesion characteristics, followed by their conformal contact onto substrates for high-quality pattern replication. Other types of materials can also be used to mediate pattern transfer, and provide opportunities to develop useful variants of $\mu \mathrm{CP} .{ }^{15,16,17}$

While many efforts in lithography are focused on improving spatial resolution and increase the complexity of pattern features, there is also a need for simple but robust micro-printing

“Corresponding author. alexwei@purdue.edu.

Supporting Information. Histogram of linewidths from 200-mesh template with parallel bars, replicated manually by MML. This material is available free of charge via the Internet at http://pubs.acs.org. 
methods with minimal requirements for infrastructure or specialized equipment. This rationale is supported by our own experiences with $\mu \mathrm{CP}$ using PDMS stamps: despite their versatility, the stamps can be sensitive to their environment and are best handled in controlled laboratory settings. In addition, the masters from which stamps are cast (typically fabricated in clean-room facilities) are delicate and must be maintained in pristine condition. The scope and accessibility of $\mu \mathrm{CP}$ can be expanded by developing "low-overhead" alternatives that can remove such practical problems in micro-printing applications, while providing comparable quality and control in pattern replication.

In this work we introduce metal-mesh lithography (MML) as an economical and overheadfree variant of $\mu \mathrm{CP}$ for reproducing microscale patterns over millimeter length scales, based on the controlled wetting of thin metal templates (Figure 1). Metallic grids of various mesh sizes are widely available as substrates for electron microscopy and have been previously employed as shadow masks in photolithography, 18,19,20,21 as well as in serigraphy (screen printing) or stencil-assisted lithography (SAL) based on plasma etching and polymerization. ${ }^{22,23,24,25}$ The patterns produced by MML, however, are inverted with respect to these techniques: ink is delivered onto the mesh template, then directly patterned onto the underlying substrate by capillary forces. In this regard, we consider MML as a hybrid of $\mu \mathrm{CP}$ and $\mathrm{CFL}$, one that requires fewer operations for pattern transfer. The metal mesh can be used repetitively for pattern replication using either commercial blotting pens or customized microquills. Binary patterns can be created by performing MML on functionalized substrates, then backfilling the ink-free regions with different materials; the MML patterns can also be removed using organic solvents (lift-off) to produce functional microarrays with well-defined edges.

\section{Experimental Section}

\section{Materials}

All chemical or biochemical reagents were obtained from Sigma-Aldrich unless otherwise noted. 3-aminopropyltriethoxysilane (APTES, 98\%) and 3-mercaptopropyltrimethoxysilane (MPTMS, 95\%) were obtained from Alfa Aesar and Fluka, respectively. Cyclo(Arg-GlyAsp-D-Phe-Lys) ( $c$-RGDfK) was purchased from Peptide International. CTAB-stabilized gold nanorods (GNRs; $\lambda_{\max }=690 \mathrm{~nm}$, O.D. 1) were prepared by a seeded growth method, ${ }^{26}$ and centrifuged once $(12500 \mathrm{~g}, 20 \mathrm{~min})$ to remove excess surfactant with redispersion in deionized water. This was obtained from an ultrafiltration system (Milli-Q, Millipore) with a measured resistivity above $18 \mathrm{M} \Omega \cdot \mathrm{cm}$ and passed through a $0.22-\mu \mathrm{m}$ filter to remove particulate matter.

Copper grids of various mesh sizes and geometries (Veco or Gilder, $3.05 \mathrm{~mm}$ diameter, 0.8 mil thickness) were obtained from Electron Microscopy Sciences: 200-mesh square lattice, hexagonal lattice, and parallel bars ( $125 \mu \mathrm{m}$ pitch, $40 \mu \mathrm{m}$ bar, $85 \mu \mathrm{m}$ spacing); 200-mesh alphanumeric index grid (125 $\mu \mathrm{m}$ pitch, $20 \mu \mathrm{m}$ bar, $105 \mu \mathrm{m}$ spacing); 400-mesh square lattice (63 $\mu \mathrm{m}$ pitch, $33 \mu \mathrm{m}$ bar, $30 \mu \mathrm{m}$ spacing); 2000 -mesh square lattice (12.5 $\mu \mathrm{m}$ pitch, 5 $\mu \mathrm{m}$ bar, $7.5 \mu \mathrm{m}$ spacing).

Glass slides were cleaned in freshly prepared piranha solution (5 parts $18 \mathrm{M} \mathrm{H}_{2} \mathrm{SO}_{4}, 2$ parts $30 \% \mathrm{H}_{2} \mathrm{O}_{2}$ ) at $70{ }^{\circ} \mathrm{C}$ for 5 minutes with stirring, then thoroughly rinsed with deionized water and dried under a stream of argon gas. Caution! Piranha solution is highly corrosive and must be handled while wearing gloves and protective clothing. Clean glass slides were immersed in a 2-mM solution of APTES in benzene or a 1-mM solution of MPTMS in toluene for $12 \mathrm{~h}$ at $25^{\circ} \mathrm{C}$. The functionalized slides were thoroughly rinsed with clean acetone to remove excess silanizing agent, then again with deionized water and dried under 
a flow of argon. NHS-functionalized glass slides (CodeLink) were obtained from SurModics and used as received.

Backfilling experiments were performed with rhodamine B isothiocyanate (RhB-ITC), fluorescein isothiocyanate (FITC), bovine serum albumin (BSA) or dye conjugates (RhBBSA or F-BSA), streptavidin from Streptomyces avidinii, Atto 550-labeled biotin, $c$ RGDfK, and CTAB-stabilized GNRs. Disposable syringes ( $3 \mathrm{~mL}$, Luer-Lok tip) and needles (22 G, 1-1/2 in) were obtained from Becton-Dickinson. Glass capillary tubes (B150-86-10) were obtained from Sutter instrument for microquill fabrication.

\section{MML patterning with 200- or 400-mesh grids}

Permanent-ink markers (Sharpie ${ }^{\circledR}$ or BiC Mark-It, ${ }^{\mathrm{TM}}$ fine point) were used as blotting pens when patterning with 200- or 400-mesh grids. In a typical print, 200-mesh copper grids ( $3.05 \mathrm{~mm}$ diameter) were positioned on functionalized glass slides using forceps, then inked with blotting pens by applying gentle pressure at the center of the template. Inking parameters were optimized using a new blotting pen, including: contact time ( 0.5 seconds), pressure $(80 \mathrm{kPa})$, tip-to-mesh contact area $\left(1.1 \mathrm{~mm}^{2}\right)$, and post-contact drying time (minimum 20 seconds), prior to removing the metal mesh template. As a practical guide for manual control over ink flow, the blotting parameters could also be tested without the metal mesh on filter paper (Whatman \#1) and adjusted to produce a $2.9-\mathrm{mm}$ round spot, slightly less than the diameter of the copper grid. It is important to minimize the inking pressure in order to prevent template adhesion and deformation during its removal from the substrate. The latter was best performed by carefully lifting one side with tweezers using a vertical motion, to prevent smudging of the newly formed pattern. All MML-patterned slides were carefully dried under ambient conditions before further use.

\section{MML patterning with 2000-mesh grids using microquills}

2000-mesh copper templates were immersed in a 1-mM solution of 1-octadecanethiol in octane for $3 \mathrm{~h}$, then washed with acetone and dried in air. MML patterning was performed using freshly pulled micro-capillaries (microquills) instead of commercial blotting pens, for more precise control over the inking volume. Microquills were prepared from glass capillary tubes $(0.86 \mathrm{~mm}$ I.D., $1.50 \mathrm{~mm}$ O.D.) using a P-87 Flaming/Brown micropipette puller (Sutter Instrument), with ramped heating optimized to produce tips with I.D. and O.D. of 35 $\mu \mathrm{m}$ and $50 \mu \mathrm{m}$, respectively. Micropipettes were cut with a ceramic tile (CTS) to produce flat tips. The microquill was then inserted inside of a 22 -gauge needle mounted on a $3-\mathrm{mL}$ plastic syringe, and sealed by wrapping wax (Parafilm) around the base.

\section{Backfilling and lift-off}

MML-patterned substrates were immersed in aqueous solutions containing fluorescent dyes or proteins in a phosphate buffered solution (PBS) adjusted to $\mathrm{pH} 8.5$ : RhB-ITC, $2 \mathrm{mg} / \mathrm{mL}$ $(3.73 \mathrm{mM}), 1 \mathrm{~h}$ at $25^{\circ} \mathrm{C}$ (APTES-functionalized slides); BSA or fluorescent BSA conjugate, $1 \mathrm{mg} / \mathrm{mL}, 20 \mathrm{~h}$ at $4{ }^{\circ} \mathrm{C}$ (CodeLink slides); streptavidin, $50 \mu \mathrm{g} / \mathrm{mL}, 48 \mathrm{~h}$ at $4{ }^{\circ} \mathrm{C} ; c$-RGDfK, $600 \mu \mathrm{g} / \mathrm{mL}(1 \mu \mathrm{M}), 12 \mathrm{~h}$ at $4{ }^{\circ} \mathrm{C}$. Streptavidin-patterned slides were labeled by treatment with Atto 550-biotin in PBS, $1 \mathrm{mg} / \mathrm{mL}$, for $2 \mathrm{~h}$ at $25^{\circ} \mathrm{C}$. MML-patterned substrates functionalized with MPTMS were treated twice with an aqueous solution of CTABstabilized gold nanorods ( $\lambda_{\max }=690 \mathrm{~nm}$; O.D. 1) by dropcasting, then drying in air.

For lift-off, slides with backfilled MML patterns were thoroughly rinsed with PBS or deionized water, then treated with reagent-grade acetone to remove the pattern and excess dye. For the best results, the lift-off procedure should be performed immediately after washing. In the case of protein-based backfills, the slides were rinsed again after lift-off with 
PBS containing $0.05 \%$ Tween-20, then again with standard PBS. In the case of dual-colored patterned slides, the lift-off step was not performed.

\section{Patterned fibroblast adhesion}

RGDfK-patterned CodeLink slides were washed repeatedly with deionized water followed by lift-off with acetone, then placed in 6-well plastic Petri dishes and incubated with GFPtransfected 3T3 fibroblasts for $12 \mathrm{~h}$ under standard cell culture conditions (RPMI medium, $37^{\circ} \mathrm{C}, 5 \% \mathrm{CO}_{2}$ ). GFP-transfected $3 \mathrm{~T} 3$ fibroblast cells were detached from the walls of a T75 tissue culture flask by treatment with trypsin, then dispensed into wells containing MML-patterned slides ( $5 \times 10^{5}$ cells/well, $2 \mathrm{~mL}$ media). Cells were grown to $80 \%$ confluence on patterned slides, which were then transferred to clean wells and washed twice with PBS (pH 7.5). The fibroblast adhesion patterns were imaged by confocal microscopy while immersed in PBS.

\section{Confocal microscopy}

All imaging studies were performed on an Olympus FV1000 laser scanning confocal microscope (inverted configuration), using a 10X or 20X objective lens for bright-field imaging. $\mathrm{RhB}$ was excited at $543 \mathrm{~nm}$ and detected using a 560-620 nm window; fluorescein and GFP were excited at $488 \mathrm{~nm}$ and detected using a 505-525 $\mathrm{nm}$ window. Confocal reflectance imaging was performed using the $635 \mathrm{~nm}$ laser line; scattered photons were detected using a 560-660 nm window. Transmission images were recorded simultaneously in all cases.

\section{Results and Discussion}

MML studies were initially performed on glass substrates using standard $\mathrm{Cu}$ electron microscopy grids (200-, 400-, and 2000-mesh) and with organic inks delivered by commercial blotting pens. Inspection of the metal grids by confocal reflectance and scanning electron microscopy revealed one face to be optically smooth and the other face rough, with grain sizes on the order of $0.3 \mu \mathrm{m}$ (Figure 2). Surface roughness has an influential role on the quality of pattern transfer: in the case of 200 -mesh grids $(125 \mu \mathrm{m}$ pitch, $40 \mu \mathrm{m}$ bar width) and 400 -mesh grids ( $63 \mu \mathrm{m}$ pitch, $33 \mu \mathrm{m}$ bar width), pattern replication is best achieved by placing the rough face of the metal mesh against the substrate. Similarly, MML with 2000-mesh $\mathrm{Cu}$ grids ( $12.5 \mu \mathrm{m}$ pitch, $5 \mu \mathrm{m}$ bar width) using the microquill delivery system (see below) can produce better quality patterns with the rough face down, which helps to limit excessive spreading.

MML is applicable to a broad range of inks and template types, and enables straightforward reproduction of structural features from square or hexagonal grids, parallel bars, and even alphanumeric characters (Figure 3). In the case of 200-mesh Cu grids (3-mm disks), uniform patterns could be replicated using commercial blotting pens with nibs of approximately 1 $\mathrm{mm}$ and a contact time and peak pressure of roughly 0.5 seconds and $80 \mathrm{kPa}$, based on tip contact area. These values are intended as practical guidelines: contact times and pressures can vary as a function of nib porosity, ink viscosity, and other environmental factors, so should be defined for each delivery system in order to avoid overpressure and partial filling of the interstitial regions. On the other hand, the uniformity of ink transfer is easily optimized by adjusting the pressure and contact area.

The quality of MML patterning is uniform across the template when the blotting pen is positioned in the center, indicating an even pressure distribution. The fidelity of lithographic reproduction can be quantified by statistical analysis: in the case of 200-mesh parallel bars ( $45 \mu \mathrm{m}$ width), the mean linewidth of the printed band patterns is $44 \pm 5 \mu \mathrm{m}(N=100$; see 
Supporting Information). The print quality is also readily apparent by the confocal imaging of fluorescent ink patterns, which clearly delineate the grid and void areas (Figure 3E,F). Lastly, the metal-mesh templates can be reused many times, with slight improvement in reproduction quality after the first few uses (Figure 4).

MML can be applied toward functionalized substrates to create periodic arrays of ink-free regions, with subsequent backfilling to produce binary patterns (Figure 5A). In the example below, a polymer-coated slide functionalized with $N$-hydroxysuccinimide (NHS) groups was subjected to MML patterning using a 200-mesh grid and red fluorescent ink, then backfilled with fluorescein-labeled BSA (F-BSA) to produce a dual-color pattern (Figure 5B,C). The primary ink layer serves as a mask that is stable under aqueous conditions, but can be easily removed by washing with organic solvents. This option is useful in situations where the backfilling materials cannot be cleanly deposited into the ink-free voids, requiring their selective removal between array elements. For example, NHS-activated slides were patterned by MML as described above, immersed in an aqueous solution containing streptavidin, then washed with acetone to produce an optically clear substrate (Figure 5D). Subsequent treatment with Atto 550-labeled biotin revealed square islands with well-defined edges after careful washing, indicating that proteins adsorbed onto the printed ink layer were removed upon lift-off (Figure 5E).

MML is also a practical method for producing functional interfaces such as microarrays of nanoparticles $27,28,29$ or ligand-based "micro-corrals" for cell patterning and adhesion. $30,31,32$ Mesh patterns were printed onto MPTMS-functionalized glass slides using a commercial blotting pen as described above, immersed for several hours in an aqueous dispersion of CTAB-stabilized Au nanorods (GNRs), then washed with acetone to reveal well-defined square lattices of GNRs (Figure 6A,B). A similar MML patterning, backfill, and lift-off procedure was performed on NHS-activated glass slides, which were treated with a cyclic pentapeptide ( $c$-RGDfK) known to promote integrin-mediated cell adhesion. ${ }^{33}$ These patterned slides were then incubated with 3T3 fibroblasts engineered to express enhanced green fluorescent protein (eGFP), and visualized by fluorescence microscopy. Fibroblast adhesion and growth was observed to be confined within each micro-corral, confirming their specificity for the RGD ligands (Figure 6C,D). In contrast, corrals functionalized with a control peptide ligand ( $c$-RADfK) did not result in patterned cell adhesion (not shown).

To determine whether MML could be performed with low micron resolution using manual control, we examined the fidelity of pattern transfer using 2000-mesh Cu grids $(12.5 \mu \mathrm{m}$ pitch, $5 \mu \mathrm{m}$ bar width). These templates are more sensitive to blotting conditions and also mechanically more fragile, and thus prone to structural defects that can adversely affect the spreading and wetting of the applied inks. Such technical issues are alleviated in part by treating the templates with chemisorptive surfactants like alkanethiols, which are well known to assemble into densely packed monolayers on metal surfaces. ${ }^{34}$ The chemisorptive coatings provide improved wetting characteristics to enable the smooth transfer of organic inks from the metal mesh onto the substrate, and also reduce the adhesion of the template to the substrate after the ink has dried.

The volume of ink used with the 200-mesh templates (ca. $0.5 \mu \mathrm{L}$ ) is excessive for MML patterning with the 2000-mesh patterns, such that the grid pattern cannot be replicated cleanly using conventional blotting pens. To address this, we developed customized microquills by pulling glass micropipettes and mounting them on syringe needles (Figure 7A). The ink is drawn into the microquills by capillary action, then delivered with precise volume control by applying hydrostatic pressure. This setup is well-adapted for dispensing customized ink formulations, which further increases the diversity of patterns that can be 
prepared by MML. In the example below, a flat-tipped glass micropipet ( $35 \mu \mathrm{m}$ I.D., $50 \mu \mathrm{m}$ O.D.) was loaded with $0.06 \mu \mathrm{L}$ of a 0.5 -wt $\%$ solution of polystyrene $(30 \mathrm{kDa})$ in $\mathrm{CHCl}_{3}$, then applied to a 2000-mesh, octadecanethiol-coated $\mathrm{Cu}$ grid placed on top of a MPTMScoated glass slide. The microquill was positioned over the center of the template and the ink was delivered manually, allowing the 2000-mesh pattern to be replicated over a finite area (ca. $0.1 \mathrm{~mm}^{2}$; Figure 7B). The polystyrene ink is also amenable to the same lift-off procedure described previously, enabling the production of GNR microarrays with island dimensions below $5 \mu \mathrm{m}$, as defined by the mesh voids (Figure 7C, D).

While the MML patterns produced by 2000-mesh grids are not as uniform as those produced by lower-mesh templates, we note that the quality and reproducibility of these higher-mesh patterns are highest when the template is prewetted with solvent, which promotes conformal contact with the substrate and smoother ink flow through capillary action. Such empirical wetting factors may be optimized by engineering the ink delivery system, an objective for further development of MML as a manual, high-definition printing technology for general use.

With respect to implementation, MML compares favorably with several other methods of microlithography (Figure 8). In CFL, the solute (ink) is first deposited onto substrates as a uniform layer by spin coating, then remolded by capillary forces induced by the differential wetting properties of the mask layer. Patterned wetting or dewetting is determined by several factors, including solvent quality of the ink, rate of solvent evaporation, and viscosity of the residual wetting layer. ${ }^{10,11} \mathrm{SAL}$ and photolithography (PL) also require the resist layer to be deposited on the substrate prior to patterning, and the range of deliverable materials is more restricted in than with other methods. $\mu \mathrm{CP}$ is the most versatile in its application, but involves separate casting and drying steps to prepare a transfer layer on the patterned surface of the stamp. In addition, the quality of material transfer is dependent on the porosity of the elastomeric stamping material and also the applied pressure, which if improperly controlled can result in stamp and pattern deformation.

MML combines inking and pattern transfer in a single step, and thus involves the fewest manipulations of all. The availability of high-quality metal templates precludes the need to fabricate and maintain expensive masters like those employed in $\mu \mathrm{CP}$, and the flexibility of manual ink delivery allows this approach to be adapted for patterning a wide variety of materials. The variables that influence MML pattern quality are similar to those encountered in CFL and $\mu \mathrm{CP}$ : the reproducibility of ink transfer is dependent on the wetting interactions and capillary forces between the substrate and metal template, and the volume of ink delivered is a function of both applied pressure and the effective retention of the blotting device (pen or microquill). On the other hand, the practical advantage of MML is the flexible combination of manual control, choice of ink composition, and minimal equipment needs, while allowing microscopic patterns to replicated across millimeter-sized areas.

\section{Conclusions}

MML is a practical "direct-write" method of microcontact printing, one that is complementary to many other templating techniques developed for micro-patterning. MML is an overhead-free alternative to CFL and elastomer-based $\mu \mathrm{CP}$ and is versatile with respect to printing conditions and ink source, and can produce functional surface patterns using simple backfilling and lift-off protocols. The ink mask can be easily removed by treatment with organic solvents, similar to the removal of negative resist layers. MML has minimum technical requirements and can be performed under manual control, with sufficient reproducibility to be useful for studies involving periodic microstructures, cell adhesion, and subsequent applications in tissue engineering $30,32,35$ and biosensing. ${ }^{36,37,38}$ 


\section{Supplementary Material}

Refer to Web version on PubMed Central for supplementary material.

\section{Acknowledgments}

The authors gratefully acknowledge financial support from the National Institutes of Health (RC1-CA147096) and the National Science Foundation (CHE-0957738). Z.T. thanks the Purdue Chemistry Department for a Stine summer fellowship. We thank David Lyvers for assistance with the pressure reader, and Prof. Greg Hockerman for the use of his micropipette puller.

\section{References}

1. Kramer S, Fuierer RR, Gorman CB. Chem. Rev. 2003; 103:4367-4418. [PubMed: 14611266]

2. Ginger DS, Zhang H, Mirkin CA. Angew. Chem. Int. Ed. 2004; 43:30-45.

3. Srituravanich W, Fang N, Sun C, Luo Q, Zhang X. Nano Lett. 2004; 4:1085-1088.

4. Chao WL, Harteneck BD, Liddle JA, Anderson EH, Attwood DT. Nature. 2005; 435:1210-1213. [PubMed: 15988520]

5. Nie ZH, Kumacheva E. Nature Mater. 2008; 7:277-290. [PubMed: 18354414]

6. del Campo A, Arzt E. Chem. Rev. 2008; 108:911-945. [PubMed: 18298098]

7. Cheng X, Guo LJ. Microelectron. Eng. 2004; 71:277-282.

8. Guo LJ. Adv. Mater. 2007; 19:495-513.

9. Suh KY, Kim YS, Lee HH. Adv. Mater. 2001; 13:1386-1389.

10. Bruinink CM, Peter M, Maury PA, De Boer M, Kuipers L, Huskens J, Reinhoudt DN. Adv. Funct. Mater. 2006; 16:1555-1565.

11. Suh KY, Park MC, Kim P. Adv. Funct. Mater. 2009; 19:2699-2712.

12. Xia Y, Whitesides GM. Angew. Chem. Int. Ed. 1998; 37:551-575.

13. Perl A, Reinhoudt DN, Huskens J. Adv. Mater. 2009; 21:2257-2268.

14. Kaufmann T, Ravoo BJ. Polym. Chem. 2010; 1:371-387.

15. Melosh NA, Boukai A, Diana F, Gerardot B, Badolato A, Petroff PM, Heath JR. Science. 2003; 300:112-115. [PubMed: 12637672]

16. Sharpe RBA, Titulaer BJF, Peeters E, Burdinski D, Huskens J, Zandvliet HJW, Reinhoudt DN, Poelsema B. Nano Lett. 2006; 6:1235-1239. [PubMed: 16771586]

17. Roca-Cusachs P, Rico F, Martinez E, Toset J, Farre R, Navajas D. Langmuir. 2005; 21:5542-5548. [PubMed: 15924487]

18. Ducker RE, Leggett GJ. J. Am. Chem. Soc. 2006; 128:392-393. [PubMed: 16402809]

19. Xu H, Hong R, Lu TX, Uzun O, Rotello VM. J. Am. Chem. Soc. 2006; 128:3162-3163. [PubMed: 16522094]

20. Xue CY, Chin SY, Khan SA, Yang KL. Langmuir. 2010; 26:3739-3743. [PubMed: 19810720]

21. Leonov AP, Wei A. J. Mater. Chem. 2011; 21:4371-4376. [PubMed: 21894240]

22. Mitchell SA, Emmison N, Shard AG. Surf. Interf. Anal. 2002; 33:742-747.

23. Malkov GS, Martin IT, Schwisow WB, Chandler JP, Wickes BT, Gamble LJ, Castner DG, Fisher ER. Plasma Proc. Polym. 2008; 5:129-145.

24. Dai LM, Griesser HJ, Mau AWH. J. Phys. Chem. B. 1997; 101:9548-9554.

25. Bullett NA, Short RD, O'Leary T, Beck AJ, Douglas CWI, Cambray-Deakin M, Fletcher IW, Roberts A, Blomfield C. Surf. Interf. Anal. 2001; 31:1074-1076.

26. Zweifel DA, Wei A. Chem. Mater. 2005; 17:4256-4261. [PubMed: 17415410]

27. Liu JF, Zhang LG, Gu N, Ren JY, Wu YP, Lu ZH, Mao PS, Chen DY. Thin Solid Films. 1998; 329:176-179.

28. Park M-H, Duan X, Ofir Y, Creran B, Patra D, Ling XY, Huskens J, Rotello VM. ACS Appl. Mater. Interfaces. 2010; 2:795-799. [PubMed: 20356283] 
29. Zheng Y, Lalander CH, Thai T, Dhuey S, Cabrini S, Bach U. Angew. Chem. Int. Ed. 2011; 50:4398-4402.

30. Roth EA, Xu T, Das M, Gregory C, Hickman JJ, Boland T. Biomaterials. 2004; 25:3707-3715. [PubMed: 15020146]

31. Gunn NM, Chang R, Westerhof T, Li G-P, Bachman M, Nelson EL. Langmuir. 2010; 26:1770317711. [PubMed: 20968293]

32. Guillotin B, Guillemot F. Trends Biotechnol. 2011; 29:183-190. [PubMed: 21256609]

33. Karp, G. Cell and Molecular Biology: Concepts and Experiments. 5th ed.. Chichester: John Wiley; 2008.

34. Laibinis PE, Whitesides GM, Allara DL, Tao YT, Parikh AN, Nuzzo RG. J. Am. Chem. Soc. 1991; 113:7152-7167.

35. Baneyx G, Baugh L, Vogel V. Proc. Natl. Acad. Sci. USA. 2002; 99:5139-5143. [PubMed: 11959962]

36. Acharya G, Chang C-L, Doorneweerd DD, Vlashi E, Henne WA, Hartmann LC, Low PS, Savran CA. J. Am. Chem. Soc. 2007; 129:15824-15829. [PubMed: 18047330]

37. Doorneweerd DD, Henne WA, Reifenberger RG, Low PS. Langmuir. 2010; 26:15424-15429. [PubMed: 20704340]

38. Adak AK, Leonov AP, Ding N, Thundimadathil J, Kularatne S, Low PS, Wei A. Bioconjugate Chem. 2010; 21:2065-2075. 

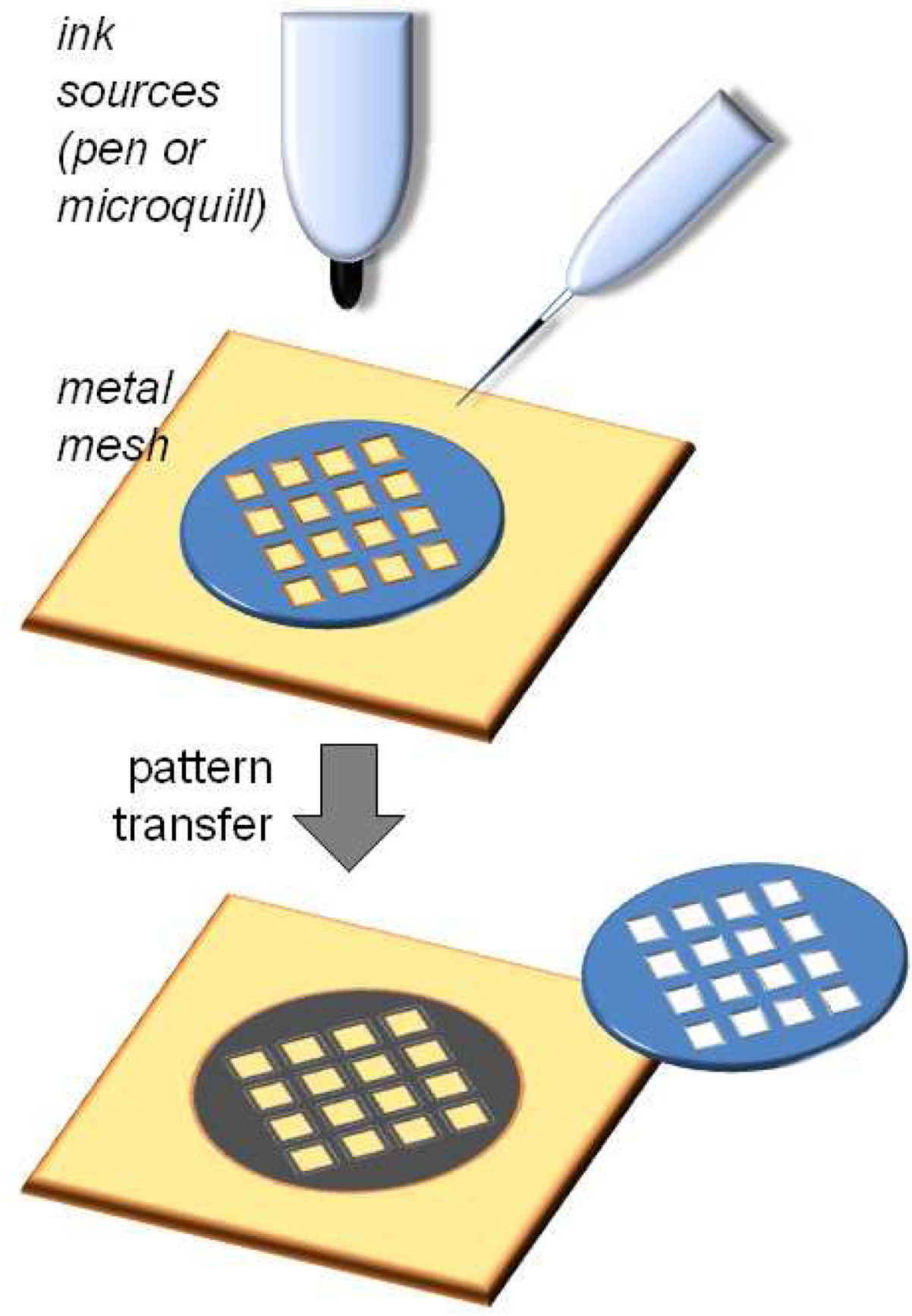

Figure 1.

Metal-mesh lithography (MML). A commercial blotting pen or customized ink delivery system (microquill) is gently pressed against a fine copper mesh on a flat surface. Controlled wetting and capillary action permits the mesh pattern to be replicated, leaving the interstitial regions untouched. 

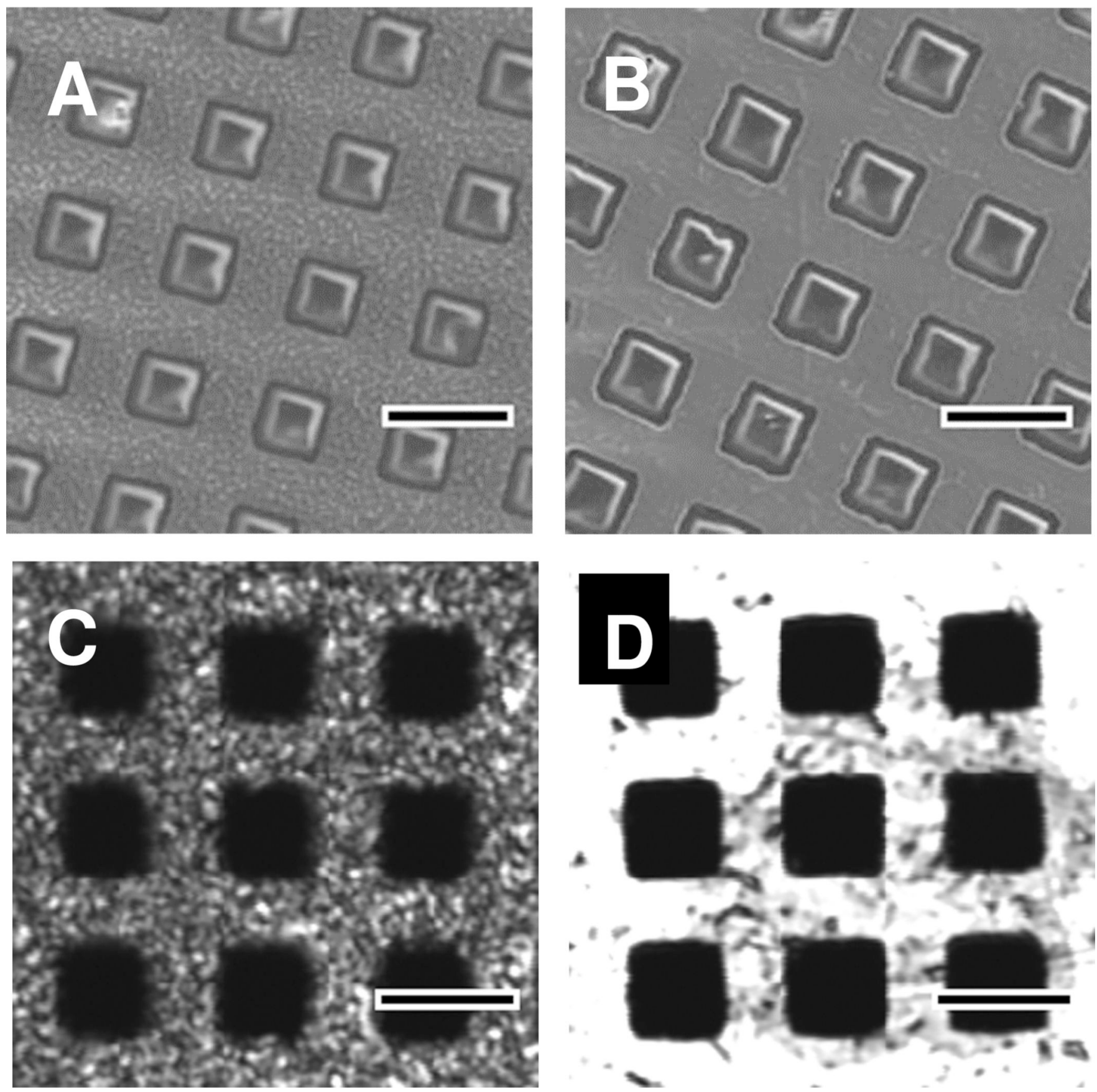

Figure 2.

Microscopic structure of 2000-mesh Cu template used in MML (plan view). (A,B) Scanning electron microscopy (SEM) and confocal reflectance images of optically rough surface; (C,D) SEM and confocal reflectance images of smooth surface using the same brightness. Scale bar $=10 \mu \mathrm{m}$. 

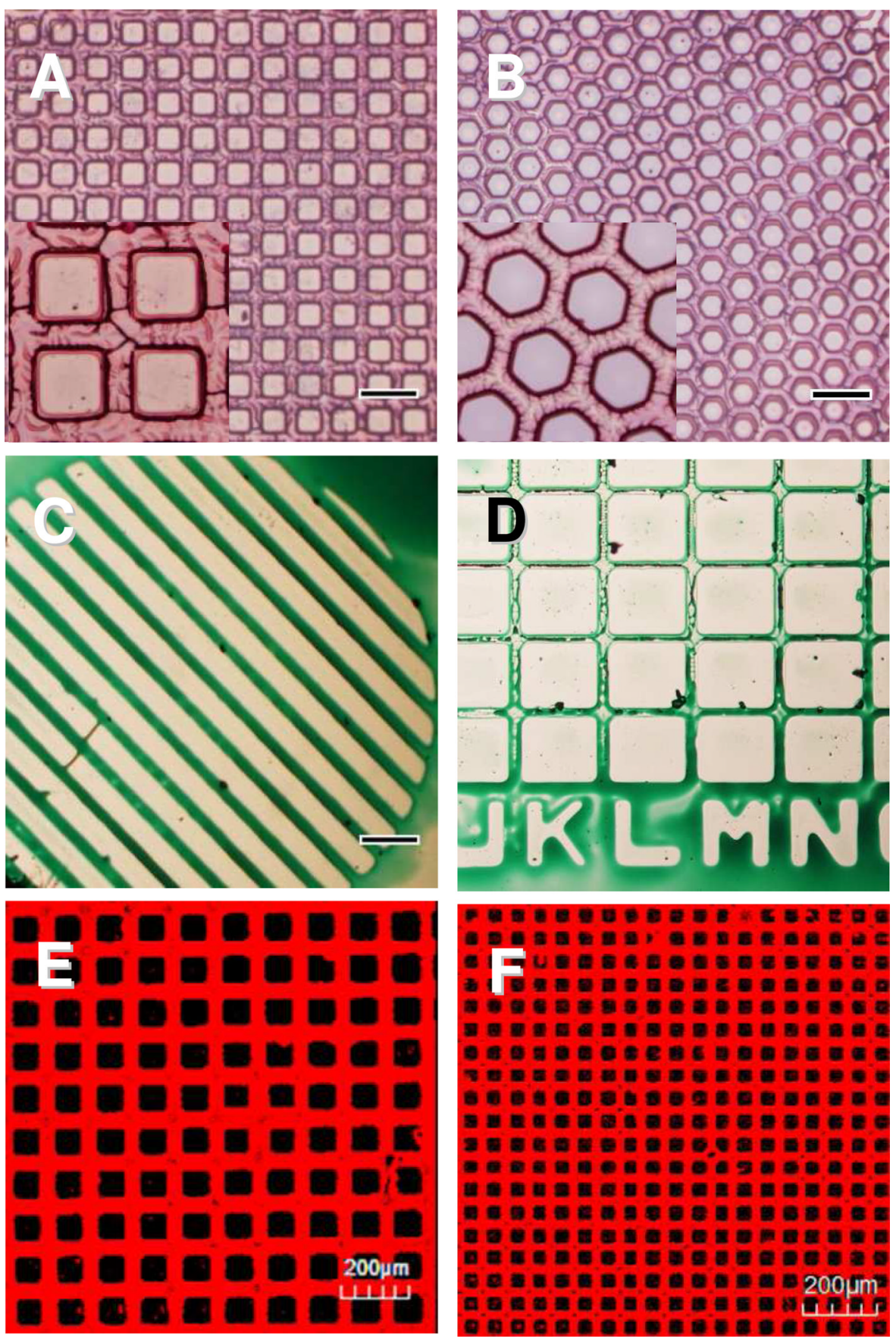

Figure 3.

(A-D) Confocal bright-field images of test patterns on glass substrates replicated by MML, using commercial blotting pens with flat tips and standard $\mathrm{Cu}$ grids and gratings. (A) 200mesh square lattice (125 $\mu \mathrm{m}$ pitch, $40 \mu \mathrm{m}$ bar); (B) 200-mesh hexagonal lattice; (C) 200mesh linear grating; (D) 200-mesh template $(125 \times 105 \mu \mathrm{m})$ with alphanumeric characters. (E,F) Confocal fluorescence images of test pattern from 200- and 400-mesh $\mathrm{Cu}$ grids $\left(\lambda_{\mathrm{ex}}=543 \mathrm{~nm}\right)$, showing a clear contrast between printed grid lines and interstitial voids. A and B produced by BiC Mark-It ${ }^{\mathrm{TM}}$ fine point (red); C and D produced by Sharpie ${ }^{\circledR}$ fine point (green); E and F produced by Sharpie ${ }^{\circledR}$ fine point (red). Scale bar $=200 \mu \mathrm{m}$. 

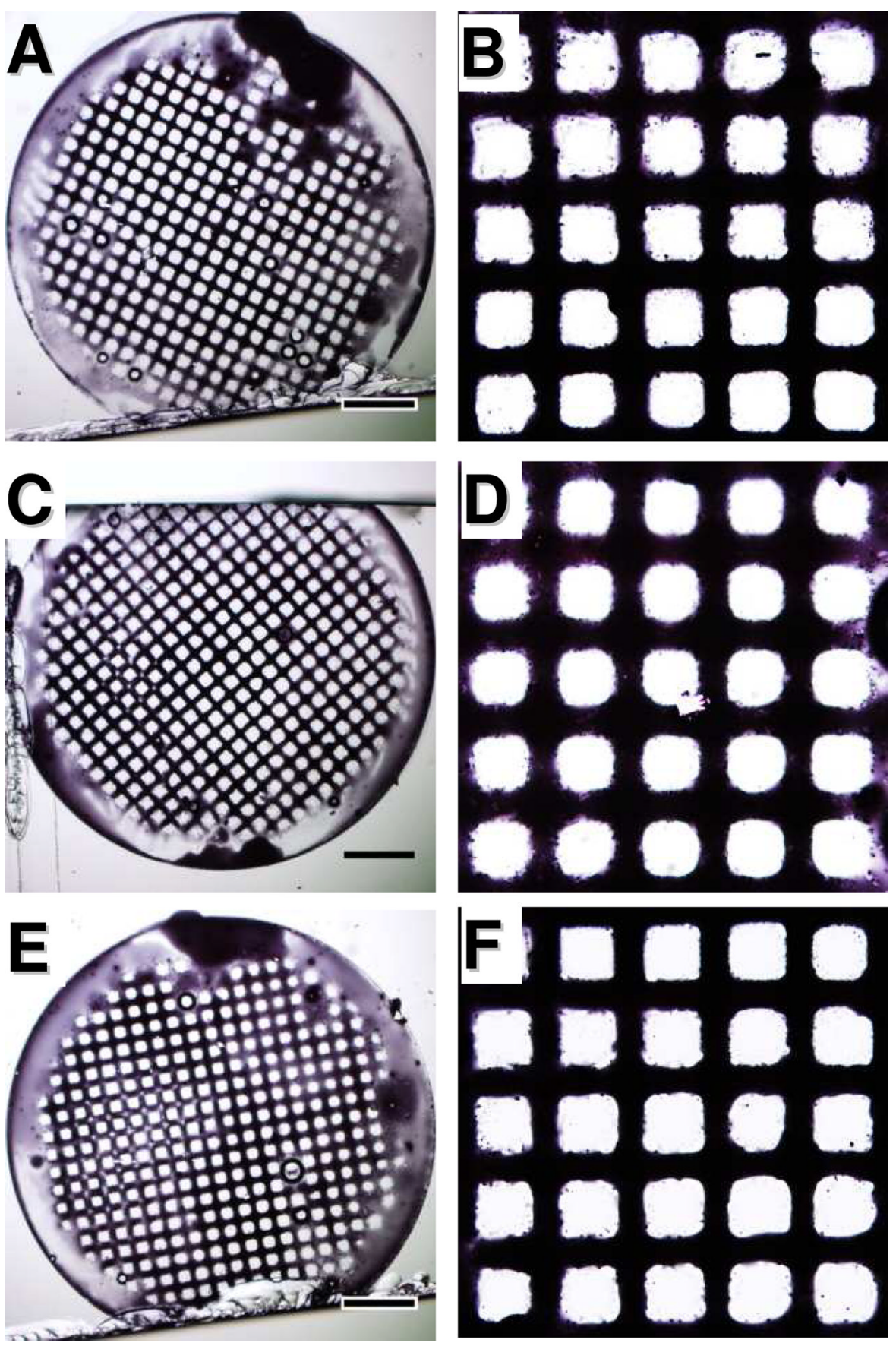

Figure 4.

Consecutive reproduction of MML patterns using the same 200-mesh $\mathrm{Cu}$ template and blotting pen (BiC Mark-It ${ }^{\mathrm{TM}}$ fine point, black). (A,B) 2nd printing; (C,D) 9th printing; (E,F) 15 th printing. Scale bar $=500 \mu \mathrm{m}$. 

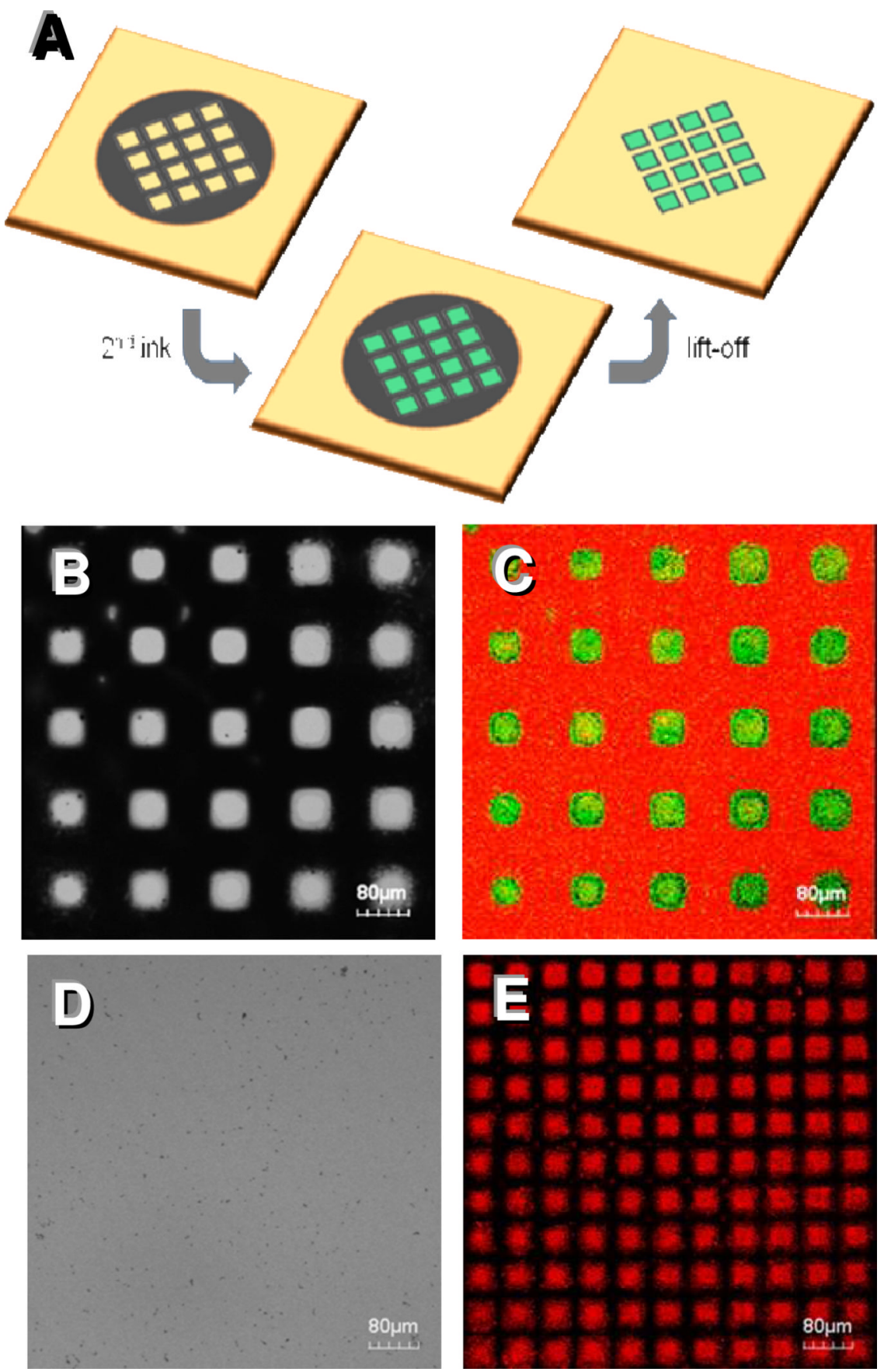

Figure 5.

(A) Formation of binary patterns and microarrays using MML and a secondary ink for the backfilling of unprinted regions. The primary ink pattern (mask) can be removed by organic solvents. (B,C) Bright-field and dual-channel fluorescence images of a 200-mesh pattern printed by MML (primary ink, red) and then F-BSA on NHS-activated (CodeLink) slides. $(\mathrm{D}, \mathrm{E})$ Bright-field and fluorescence images of streptavidin microarray (labeled with Atto 550-biotin) after lift-off of a 400-mesh pattern mask. 

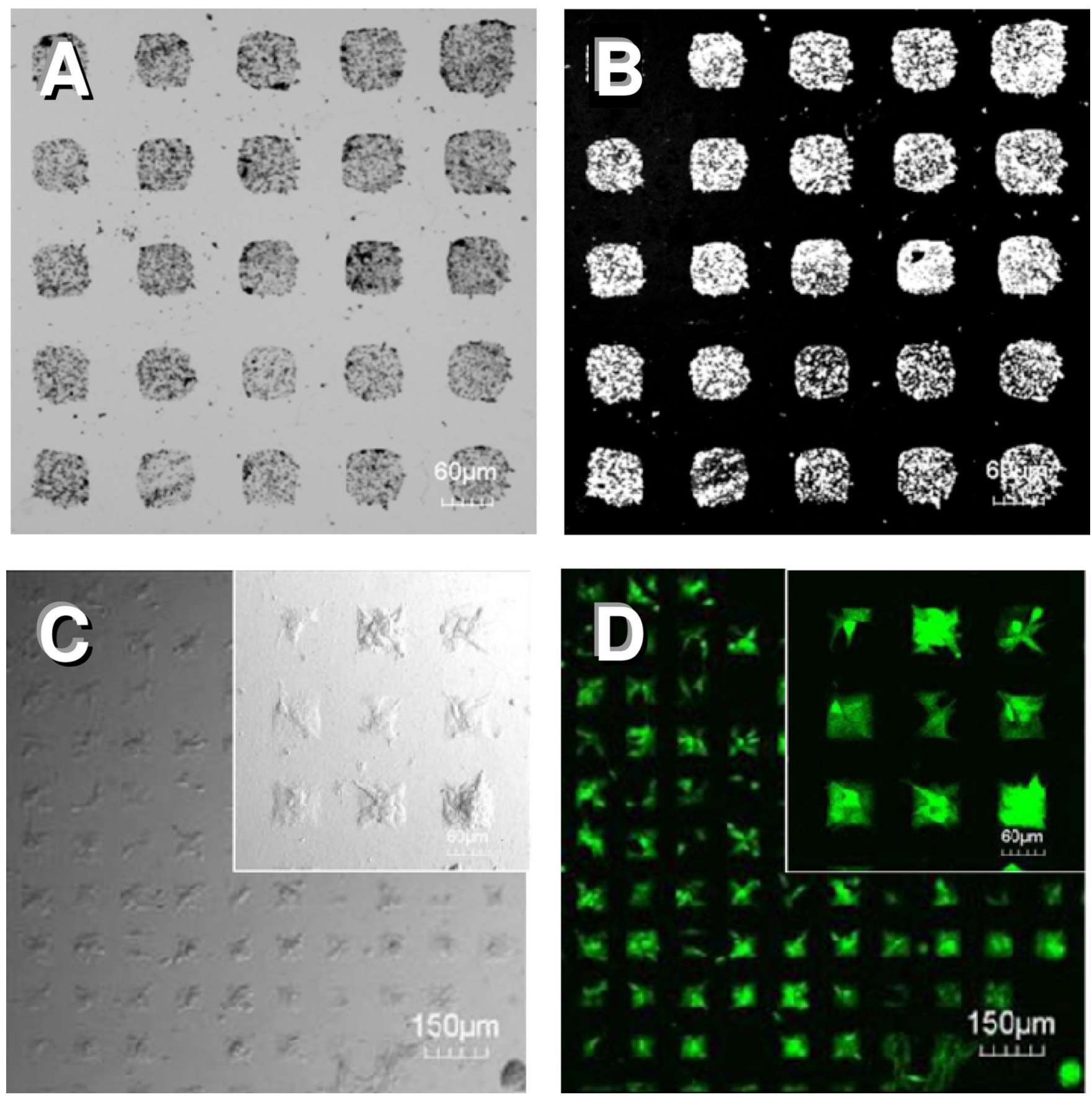

Figure 6.

(A,B) Bright-field and confocal reflectance images of GNR microarrays on an MPTMStreated glass slide, prepared in three steps: (i) MML using a 200-mesh template, (ii) electrostatic adsorption of GNRs; (iii) lift-off of primary ink pattern. (C,D) Bright-field and fluorescence images of eGFP-producing 3T3 fibroblasts in micro-corrals, functionalized with $c$-RGDfK for integrin-mediated cell adhesion. 

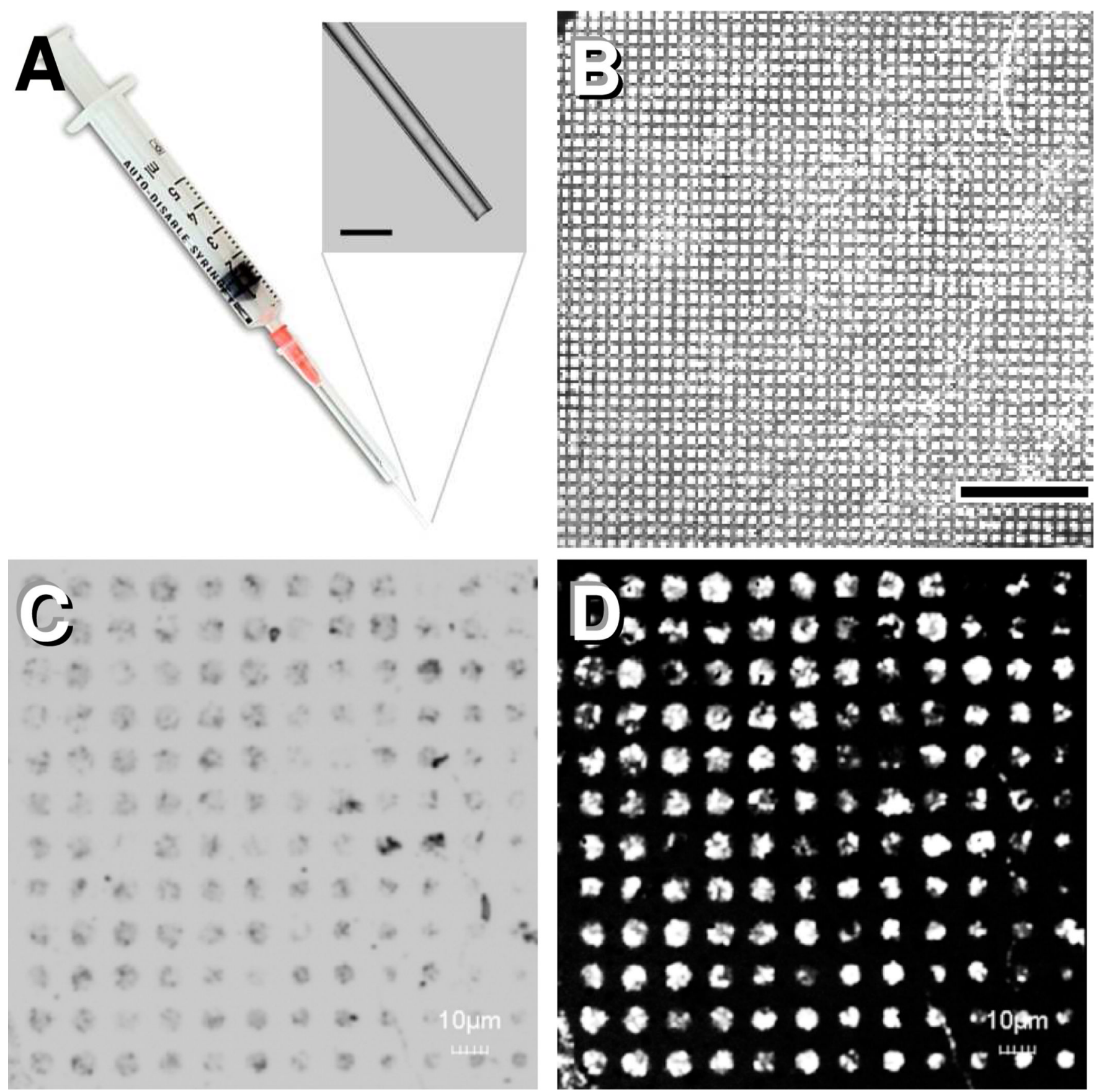

Figure 7.

MML patterning using a 2000-mesh Cu grid passivated with octadecanethiol. (A) Schematic of microquill for delivery of customized ink ( $0.5 \mathrm{wt} \%$ polystyrene in $\left.\mathrm{CHCl}_{3}\right)$; inset, confocal image of microquill tip (scale bar $=100 \mu \mathrm{m}$ ). (B) Confocal bright-field image $(\lambda=635 \mathrm{~nm})$ of 2000-mesh pattern with well-shaped square voids, replicated manually by MML. (scale bar $=100 \mu \mathrm{m})(\mathrm{C}, \mathrm{D})$ Bright-field and confocal reflectance images of GNR microarray on MPTMS-coated slides, after lift-off of MML-generated ink pattern. 


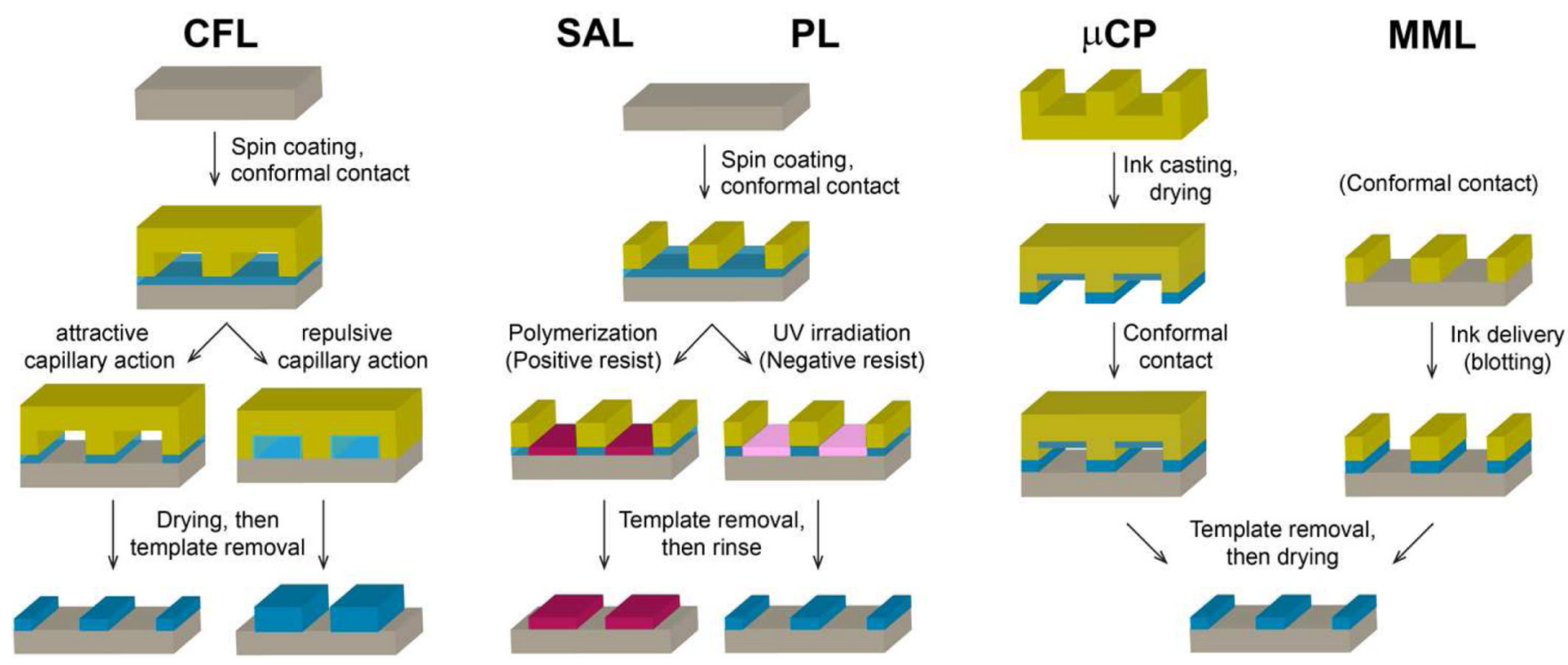

Figure 8.

Stepwise procedures for various lithographic methods, with substrate in grey, template in yellow, and solute (ink) in blue. Left to right: capillary force lithography (CFL); stencilassisted lithography (SAL) and photolithography (PL); microcontact printing ( $\mu \mathrm{CP})$; metalmesh lithography (MML). For SAL/PL, positive and negative resists after exposure are dark and light red, respectively. 